\title{
Hipercetonemia: bioquímica de la producción de ácidos grasos volátiles y su metabolismo hepático
}

\section{Hyperketonemia: Biochemistry of volatile fatty acid production and its hepatic metabolism}

\author{
Oscar Felipe Huertas-Molina ${ }^{1}$; Daniela Londoño-Vásquez ${ }^{2}$; Martha Olivera-Angel ${ }^{3}$
}

\begin{abstract}
'MVZ. M.Sc. Corporación Colombiana de Investigación Agropecuaria, AGROSAVIA, Centro de Investigación El Nus. San José del Nus, Antioquia, Colombia; e-mail: ohuertas@agrosavia.co; (D) https://orcid.org/0000-0001-9021-8115
\end{abstract}

${ }^{2}$ MV. M.Sc. Universidad de Antioquia, Grupo de investigación Biogénesis. Medellín - Antioquia, Colombia; e-mail: daniela.londonov@udea.edu.co; (D) https://orcid.org/0000-0002-2537-491X

${ }^{3}$ DMV, Ph.D. Universidad de Antioquia, Facultad de Ciencias Agrarias, Grupo de investigación Biogénesis. Medellín - Antioquia, Colombia; e-mail: martha. olivera@udea.edu.co; (D) https://orcid.org/0000-0001-7853-4406

\begin{abstract}
Cómo citar: Huertas-Molina, O.F.; Londoño-Vásquez, D.; Olivera-Angel, M. 2020. Hipercetonemia: bioquímica de la producción de ácidos grasos volátiles y su metabolismo hepático. Rev. U.D.C.A Act. \& Div. Cient. 23(1):e1304. http://doi.org/10.31910/rudca.v23. $\mathrm{n} 1.2020 .1304$

Artículo de acceso abierto publicado por Revista U.D.C.A Actualidad \& Divulgación Científica, bajo una licencia Creative Commons CC BY-NC 4.0

Publicación oficial de la Universidad de Ciencias Aplicadas y Ambientales U.D.C.A, Institución de Educación Superior Acreditada de Alta Calidad por el Ministerio de Educación Nacional.

Recibido: Julio 25 de 2019

Aceptado Mayo 27 de 2020

Editado por: Ingeborg Zenner de Polanía
\end{abstract}

\section{RESUMEN}

La hipercetonemia o cetosis bovina es un desorden metabólico, que se caracteriza por el incremento patológico de cuerpos cetónicos (beta-hidroxibutirato ( $\beta \mathrm{HB}$ ), Acetoacetato (AcAc) y acetona) y ocurre en el periparto de vacas de leche. El origen primario de la enfermedad es el balance energético negativo (BEN), que puede ser desencadenado por el incremento excesivo de los requerimientos energéticos o la presentación de enfermedades posparto, resultando en la presentación de signos clínicos o disminución de la producción de leche. El objetivo de esta revisión consiste en describir, mediante un modelo, los procesos bioquímicos del rumen y los mecanismos fisiopatológicos, involucrados con incremento excesivo de los cuerpos cetónicos. En resumen, se realizó un modelo fisiológico uniendo literatura fragmentada, sobre la relación entre la función ruminal, hepática y la inducción de lipolisis e incremento de la actividad de Carnitil-Palmitoil transferasa-1 (CPT-1), cuyo resultado puede ser la producción excesiva de Acetil-CoA que, junto con la falta de propionato y oxalacetato (precursores de gluconeogénesis y ciclo de Krebs), dan lugar a la producción patológica de acetoacetato y beta-hidroxibutirato.

Palabras clave: balance energético negativo; cetosis; cuerpos cetónicos; vaca lechera.

\section{ABSTRACT}

Bovine hyperketonemia or ketosis is a metabolic disorder characterized by high levels of ketone bodies (beta-hydroxybutyrate $(\beta \mathrm{HB})$, Acetoacetate (AcAc), and acetone) in periparturient dairy 
cows. A Negative Energy Balance (NEB) is identified as the primary cause of the disease, which is triggered by the excessive increase of energy requirements or the presence of postpartum diseases, resulting in the appearance of clinical signs or decreased milk production. The purpose of this review is to describe the rumen's biochemical Process and the physiopathological mechanisms involved in the excessive production of ketone bodies. After conducting a literature review, a physiological model was carried out in order to understand the relationship between the rumen and liver functions with lipolysis induction and increased CPT-1 activity. The above may result in the overproduction of Acetyl-CoA, which together, with the lack of propionate and oxaloacetate (gluconeogenesis and Krebs cycle precursors), leads to the pathological production of acetoacetate and beta-hydroxybutyrate.

Keywords: negative energy balance; ketosis; ketone bodies; dairy cattle.

\section{INTRODUCCIÓN}

La cetosis (Garzón \& Oliver, 2018), acetonemia (MadresehGhahfarokhi et al. 2018) o hipercetonemia (Duffield et al. 2009; Mann et al. 2017) bovina, se ha definido como la concentración elevada de cuerpos cetónicos (Acetoacetato, Beta-hidroxibutirato (BHB) y Acetona) circulantes (Chandler et al. 2017). Esta condición puede afectar negativamente la salud del animal, por la presentación de signos clínicos de cetosis y por la asociación que esta tiene con enfermedades puerperales, como desplazamiento de abomaso, metritis y claudicaciones (Duffield et al. 2009). Se ha observado también, que en animales con cetosis subclínica, la producción de leche disminuye significativamente (Xu et al. 2017, Antanaitis et al. 2018). El $\beta$-hidroxibutirato ( $\beta$ HB), uno de los cuerpos cetónicos, se usa para diagnosticar esta condición, por ser el más predominante y estable en la leche, la orina y el suero

En condiciones fisiológicas normales, se producen cuerpos cetónicos, que son los que proveen de energía a los tejidos extrahepáticos y su concentración no debe superar $1.2 \mathrm{mmol} / \mathrm{Lt}$, en sangre o $0.15 \mathrm{mmol} /$ Lt, en leche (Duffield et al. 2009; Chandler et al. 2017). La razón, por la cual, se da un aumento en la concentración es porque las demandas de energía sobrepasan la capacidad de consumo de carbohidratos y fallan los mecanismos de adaptación contra el incremento de niveles de insulina (resistencia a la insulina), lo que es denominado balance energético negativo (BEN) (Herdt, 2000; Friggens et al. 2007), caracterizado por el metabolismo de triglicéridos que, a su vez, generan el aumento en circulación de los cuerpos cetónicos (Langin, 2006; Smith, 2013).

Los mecanismos fisiológicos y patológicos de producción de cuerpos cetónicos, durante el periodo de transición de la lactancia en bovinos, están explicados de manera dispersa y fragmentada en la literatura, por lo tanto, el objetivo de este modelo es integrar el conocimiento de los eventos bioquímicos fisiológicos y cómo durante el posparto esta producción se puede tornar patológica y conducir a la hipercetonemia.

\section{MATERIALES Y METODOS}

Para llevar a cabo esta revisión, se tomaron artículos científicos entre 1974 y 2019, de bases de datos, como PUBMED, Science Direct, Scopus y Google académico. Mediante el análisis de estos artículos, se propone el modelo metabólico de cetogénesis, a partir de la fisiología del rumen, hígado y tejido adiposo.

Esta revisión, se realizó entre 2018 y 2019 y analizó más de 50 referencias, publicadas en los últimos 35 años, seleccionadas a criterio de los autores.

\section{RESULTADOS Y DISCUSIÓN}

Modelo metabólico de cetogénesis a partir de la fisiología del rumen, hígado y tejido adiposo. Consiste en la agrupación de rutas metabólicas que tienen en común la síntesis de cuerpos cetónicos, bajo condiciones fisiológicas del posparto temprano de hembras bovinas lecheras. Estas rutas están relacionadas con la producción, la absorción y el metabolismo de los Ácidos Grasos Volátiles (AGV), lipolisis del tejido adiposo y beta oxidación de Ácidos Grasos No Esterificados (AGNES), en los hepatocitos (Engelking, 2015). Después de la cetogénesis, como subproducto de estas rutas, los cuerpos cetónicos pueden ser usados por tejidos extrahepáticos, como fuente de Acetil CoA o incrementar sus niveles circulantes a patológicos, generando cetosis clínica o subclínica (Langin, 2006; Smith, 2013).

\section{Bioquímica bacteriana de la producción de Ácidos Grasos} Volátiles (AGV) en el rumen. La masticación y el proceso de rumia consisten en fraccionar el alimento, en este caso, el forraje verde, convirtiéndolo en partículas más pequeñas, para que sea más fácil su digestión, por parte de las bacterias (Millen et al. 2016). Durante este proceso, el forraje degradado por la rumia, se queda en el rumen; en este órgano, la población bacteriana presente (entre $10^{10}$ y $10^{11}$ bacterias/gramo de contenido ruminal) metaboliza el sustrato y excreta AGV. La población microbiana del rumen está conformada, en su mayoría, por las bacterias celulolíticas, amilolíticas y sacarolíticas que, entre otros metabolitos, producen acetato, propionato y butirato, en diferentes concentraciones (Pabon, 2004); el ejemplo de este modelo (Figuras 1 y 2), se basa en el metabolismo de las bacterias celulolíticas, que producen acetato $(70 \%)$, propionato $(20 \%)$ y butirato $(10 \%)$. Estas bacterias son las que se encuentran en mayor cantidad en el forraje; este último, está conformado, principalmente, por celulosa, hemicelulosa y, en menor proporción, por lignina, pectina y lípidos (Church, 1993; Vaidya et al. 2019).

Las bacterias celulolíticas metabolizan la celulosa por medio de la acción del complejo celulasa extracelular (CCE), que está compuesto por varias enzimas: la exoglucanasa, la endoglucanasa y la $\beta$-glucosidasa (Pratama et al. 2014). Las dos primeras, actúan en la hidrólisis escalonada de la celulosa (polisacárido) hasta celobiosa (disacarido); posteriormente, la $\beta$-glucosidasa, hidroliza la celobiosa a dos moléculas de glucosa (monosacaridos) (Ratanakhanokchai 


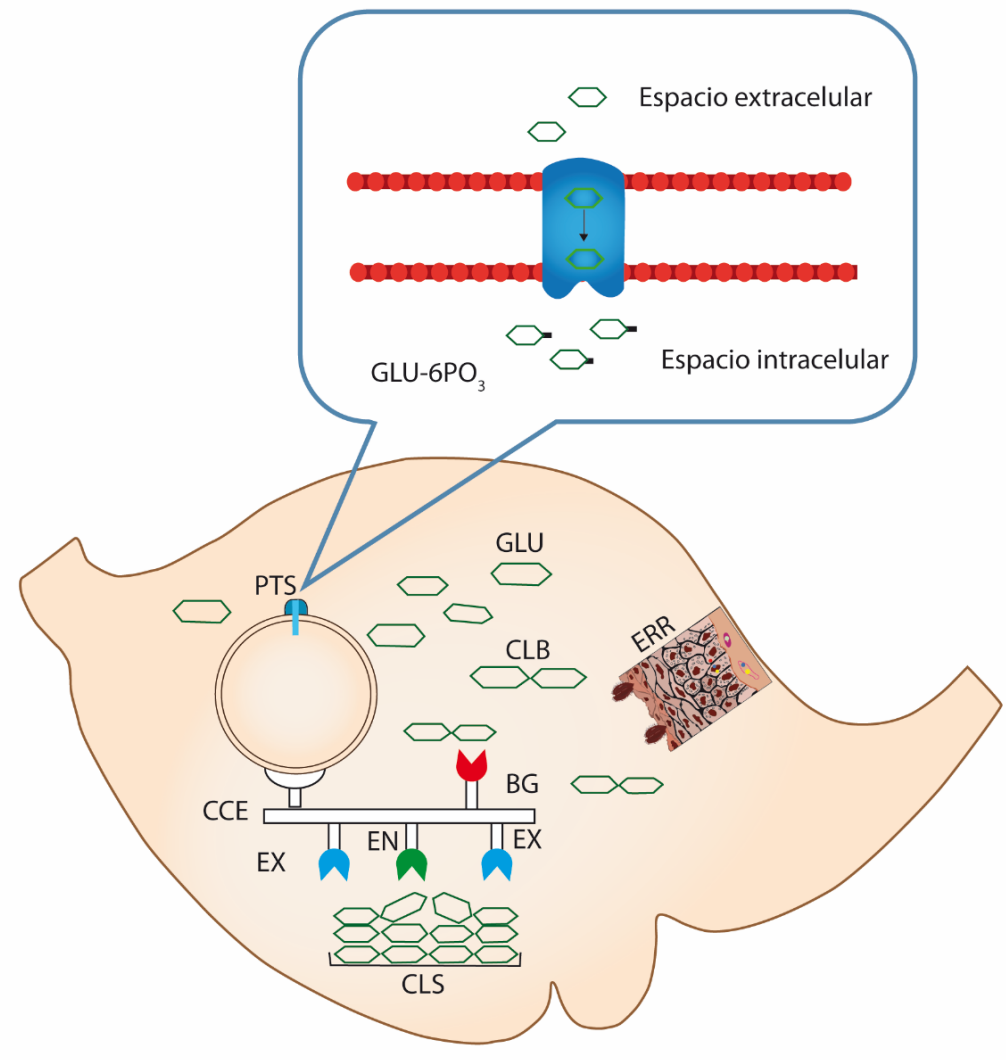

Figura 1. Degradación de la celulosa y obtención de glucosa por parte de la bacteria celulítica ruminal. ERR: Epitelio Ruminoreticular; GLU: Glucosa; CLB: Celobiosa; BG: $\beta$-glucosidasa; EX: Exoglucanasa; EN: Endoglucanasa; CLS: Celulosa; GLU-6PO3: Glucosa-6-fosfato. Adaptado de Ratanakhanokchai et al. (2013) y Baldwin \& Connor (2017).

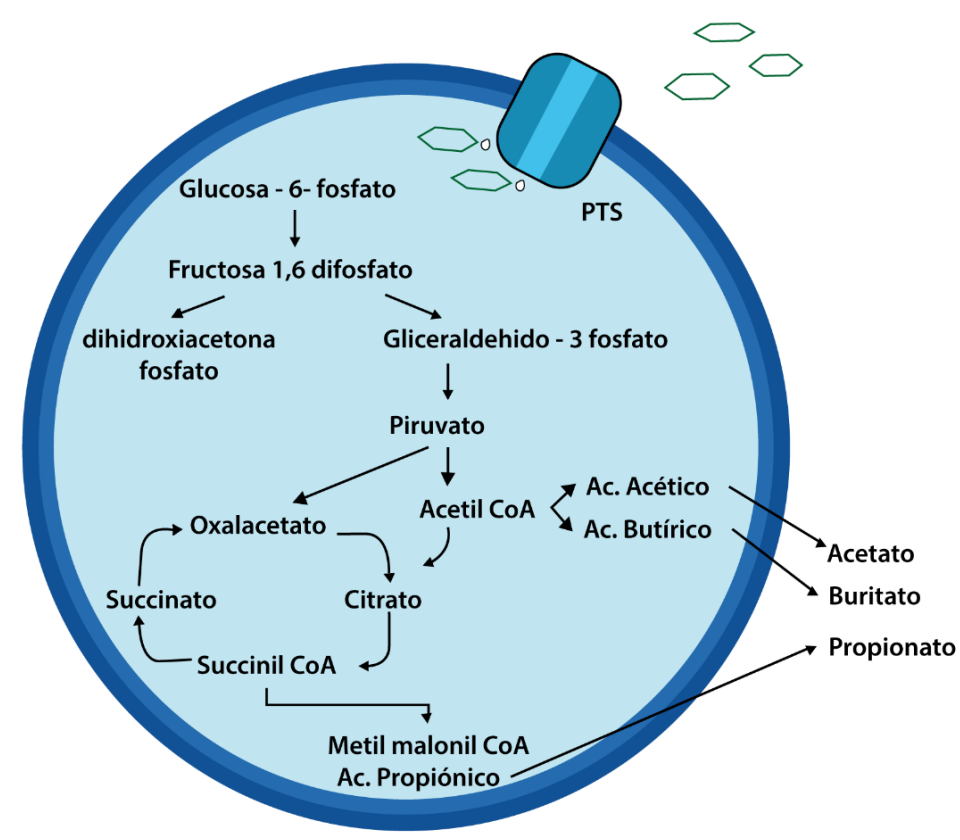

Figura 2. Bacteria celulolítica ruminal: Resumen de la glicolisis, rama dicarboxilica del ciclo de Krebs y ciclo de Wood-werkman para la producción de Acetato, butirato y propionato. 
et al. 2013) (Figura 1). Por un canal proteico, llamado sistema fosfotransterasa (PTS), la glucosa ingresa unida a un fosfato, para producir glucosa-6-fosfato que, posteriormente, ingresa al citoplasma de la bacteria, a partir, de la cual, inicia la glicólisis y, su producto final, son dos moléculas de piruvato (Franklundt \& Glass, 1987; Jiang et al. 2014). El PTS, también puede transportar celobiosa hacia el interior de la bacteria (Figura 1) (Maas \& Glass, 1991; Ratanakhanokchai et al. 2013; Jiang et al. 2014).

El proceso de fermentación de la glucosa en el rumen por parte de las bacterias celulíticas es anaeróbico y la relación de acetato, propionato y butirato, producidos cuando la dieta se basa en forraje, es 70:20:10, respectivamente (Smith, 2013).

La glicólisis bacterial se da en tres etapas: la primera o etapa preparativa, en la que hay consumo de energía sin óxidoreducción, donde el ATP se usa para fosforilar los precursores de dihidroxiacetona fosfato (DHA) y gliceraldehido-3-fosfato (G-3P) (Church, 1993).

La segunda etapa, se caracteriza por la liberación de energía en forma ATP, a partir de la óxido-reducción del G-3P, que termina con la formación de dos moléculas de piruvato (Millen et al. 2016). En este momento, inicia la tercera etapa: el piruvato se puede carboxilar por acción de la enzima piruvato carboxilasa y producir oxaloacetato, que ingresa al ciclo de Krebs; va a la formación de succinil-CoA que, por acción de la metimalonil mutasa es convertido en metilmalonil- CoA, precursor del ácido propiónico (Millen et al. 2016; Baldwin \& Connor, 2017). El piruvato, también se puede descarboxilar por acción de la enzima piruvato deshidrogenasa y producir Acetil-CoA, precursor del ácido acético y ácido butírico (Figura 2) (Vital et al. 2014).

Para la formación de acetato, el Acetil-CoA es convertido en Acetilfosfato por la enzima fosfo-transacetilasa, que permite el clivaje de un grupo CoA y la unión de un átomo de fosforo al radical acetil. Este es catalizado por la enzima acetato quinasa, que permite la liberación de una molécula de ATP y da lugar al ácido acético (Figura 2) (White et al. 2012; Hackmann et al. 2017).

Para la formación de butirato, el Acetil-CoA, por medio de la enzima tiolasa, se transforma en acetoacetil-CoA, que continúa con reacciones de óxido reducción hasta llegar a butiril-CoA (Pabon, 2004; Wang et al. 2020); a este compuesto, la fosfo-trans butirilasa le cliva un grupo CoA y le adhiere un átomo de fósforo, para que la butirato quinasa, actúe sobre el butiril-fosfato, facilitando la liberación de ATP, dando lugar al ácido butírico (Vital et al. 2014; Hackmann \& Firkins, 2015).

La producción de propionato es a partir del piruvato que, por la enzima Metil malonil-CoA que lo carboxila, produce oxalacetato, que ingresa por la rama dicarboxilica (Oxalacetato-malato-fumaratosuccinil -CoA), del ciclo de Krebs y produce succinil CoA (Engelking, 2015; Nielsen et al. 2017). La Metil Malonil CoA, simultáneamente, se produce en el ciclo de Wood-Werkman, cuando actúa sobre el piruvato y se convierte en propionil CoA que, a su vez, actúa sobre el succinil CoA y produce propionato (Nielsen et al. 2017). Esta vía de producción de propionato es autolimitante porque depende de la producción de oxaloacetato, el cual, es clave para los ciclos Wood-Werkman y Krebs (Liu et al. 2015).

El acetato, el propionato y el butirato corresponden a la forma disociada del producto final de la bacteria, que es Ácido acético, Ácido propiónico y Ácido Butírico, con lo cual, se pueden absorber en la pared dorsal del rumen, aunque los AGV no disociados también se pueden absorber por el rumen (Dijkstra et al. 2012).

Metabolismo de los Ácidos Grasos Volátiles. Los AGV absorbidos por la pared dorsal del rumen (acetato, butirato y propionato) son la forma disociada de los ácidos grasos producidos por la fermentación bacteriana (ácido acético, ácido butírico y ácido propiónico) (Figura 2) (Storm et al. 2012; Van Lingen et al. 2016; Baldwin \& Connor, 2017). Se ha comprobado que los AGV pueden ingresar al epitelio ruminal por difusión simple y mediante mecanismos de difusión, facilitada a través de transportadores de monocarboxilatos (MCT-1, MCT-2 y MCT-4) e intercambiadores de sodio/protones (Koho et al. 2005; Kirat et al. 2006; 2007; Nakamura et al. 2018); además, se ha comprobado de los AGV inducen la expresión de estos transportadores (Nakamura et al. 2018).

Más del 50 - 75\% de los AGV son absorbidos, a través del epitelio rumino-reticular (ERR) (Harfoot, 1981), principalmente, por las células del estrato basal (Church, 1993; Baldwin \& Jesse, 1991); los AGV restantes $(25-50 \%)$, se absorben en el omaso (Pratti Daniel et al. 2006; Pratti Daniel \& Resende Júnior, 2012) e intestino. Las células del ERR tienen mitocondrias altamente funcionales, donde hay compartamentalización de enzimas cetogénicas, que contribuyen significativamente a la producción de Aceto-acetato y $\beta$-hidroxibutirato (cuerpos cetónicos) (Leighton et al. 1983).

Ruta cetogénica del butirato y del acetato. El epitelio ruminal es el principal productor de cuerpos cetónicos en el rumiante. Cerca del 85 al 90\% del butirato es absorbido y metabolizado inicialmente por las células basales del rumen (Baldwin, 1998). Después del ingreso por difusión simple o a través de transportadores de monocarboxilatos'T (1, 2 o 4) (Nakamura et al. 2018), la enzima Butiril-Coa sintetasa, ubicada en el citoplasma, activa el butirato, adicionándole un grupo CoA (Webster et al. 1965). El transporte intracelular del butirato no está claro; sin embargo, el transporte activo por MCT-1, la difusión simple o el transporte por la unión a un grupo CoA permiten la entrada del butiril $\mathrm{CoA}$ a la mitocondria. Una vez dentro de la organela, el butiril CoA pasa por $\beta$-oxidación y es convertido en acetoacetil CoA (AcAc CoA) (Emmanuel, 1980; Emmanuel \& Milligan, 1983). La enzima acetoacetil CoA tiolasa 1 (ACAT1) puede clivar el AcAc-CoA para producir Acetil CoA y ser usado en el ciclo de Krebs (Kato et al. 2015); no obstante, las enzimas del epitelio ruminal hacen que los cuerpos cetónicos se puedan producir de dos maneras: la primera, puede progresar a través del 3-hidroxi-3-metilglutaril CoA sintasa (HMG-CoA sintasa) y 3-hidroxi-3-metilglutaril CoA liasa (HMG CoA liasa) (como en las células hepáticas) o, por desacetilación de Acetoacetil CoA, catalizado por succinil CoA transferasa. Cualquier de las 
dos vías producen AcetoAcetato (AcAc) (Heitmann et al. 1987). $\mathrm{El}$ paso final en la cetogénesis ruminal es la producción de $\beta \mathrm{HB}$, que es catalizado por la enzima $\beta$-hidroxibutirato deshidrogenasa ( $\beta$ HB-DH) (Bruss, 2008); en el bovino, esta vía es favorecida por la relación NADH:NAD, existente en las mitocondrias del epitelio ruminal (Figura 3) (Heitmann et al. 1987).

La mayor parte del acetato es utilizado, principalmente, en la síntesis de grasa en el tejido adiposo y como sustrato energético en tejidos extrahepáticos (Kristensen et al. 2005; Herdt, 2000). El acetato, también ingresa por difusión simple o a través de transportadores MCT (1 y 4) (Aluwong et al. 2010), al citoplasma de las células blanco; posteriormente, interactúa con la enzima Acetil CoA sintetasa y se convierte en Acetil CoA (Pabon, 2004; Xiang et al. 2016).

En el adipocito, el Acetil CoA citoplasmático marca el inicio de la lipogénesis por la vía malonil CoA, resultando en la formación de triglicéridos y colesterol (Lipogenesis); esta vía es dependiente de la activación de la enzima Acetil CoA carboxilasa (Matsumoto et al. 2012).



Figura 3. Vías metabólicas de los Ácidos Grasos Volátiles cetogénicos. LB: Lámina Basal; EB: Estrato Basal; EE: Estrato Espinoso; EG: Estrato Granuloso; EC: Estrato Córneo; VS: Vasos Sanguíneos de la lámina basal del epitelio ruminal; MAG: Mono-Acil-Glicerol; DAG: Di-Acil-Glicerol. Adaptado de: Baldwin \& Connor, 2017.

En células de tejidos extrahepáticos, como corazón, músculo esquelético, riñón y glándula mamaria, el acetato es utilizado como fuente de energía, a partir de la enzima Acetil CoA sintetasa, que lo convierte en Acetil CoA en el citoplasma o en la mitocondria, para ser utilizado en el ciclo de Krebs (Figura 2) (Pabon, 2004; Xiang et al. 2016).

Ruta glucogénica. En el epitelio ruminal, cerca del 3 - 15\% del propionato es metabolizado a lactato y piruvato (Weigand et al. 1975; Nocek et al. 1980; Emmanuel, 1981; Baldwin \& Jesse, 1996). Posteriormente, es transportado por circulación portal al hepatocito, donde es carboxilado por la enzima Propionil-CoA carboxilasa, que se encuentra en el citoplasma de la célula (Wongkittichote et al. 2017). Una vez activado (que es la adición de un grupo CoA), el propionil-CoA ingresa a la matriz mitocondrial y es convertido en metil-malonil-CoA, por el complejo de proteínas B12, a succinil CoA, que ingresa al ciclo de Krebs y sirve para la producción de oxalacetato. El oxalacetato sale al citoplasma desde la mitocondria, por medio del mecanismo de lanzadera mitocondrial. Con éste, se da inicio a la gluconeogénesis por la vía Fosfoenol piruvato (PEP) (Figura 4) (Watford et al. 1981; Aschenbach et al. 2010; White et al. 2012; Engelking, 2015).

Metabolismo lipolítico. La partición de nutrientes en el posparto está relacionada con el balance energético negativo y la habilidad que tiene la vaca para compensar éste, mediante la movilización de productos del tejido graso. El déficit es causado por el incremento de los requerimientos de energía para la producción de leche $\mathrm{Xu}$ et al. 2018),

El recurso energético extra lo saca del tejido graso, a través de la lipolisis, que consiste en la hidrólisis de Triglicéridos (TAG) por la lipoproteína lipasa, que deja, como resultado, un grupo glicerol y ácidos grasos no esterificados (AGNE's), que pueden ser de cadena larga o media, los cuales, son transportados por la albúmina hacia el hepatocito, en donde el AGNE ingresa por difusión pasiva o facilitada, luego de ser liberado de su transportador (Murray et al. 


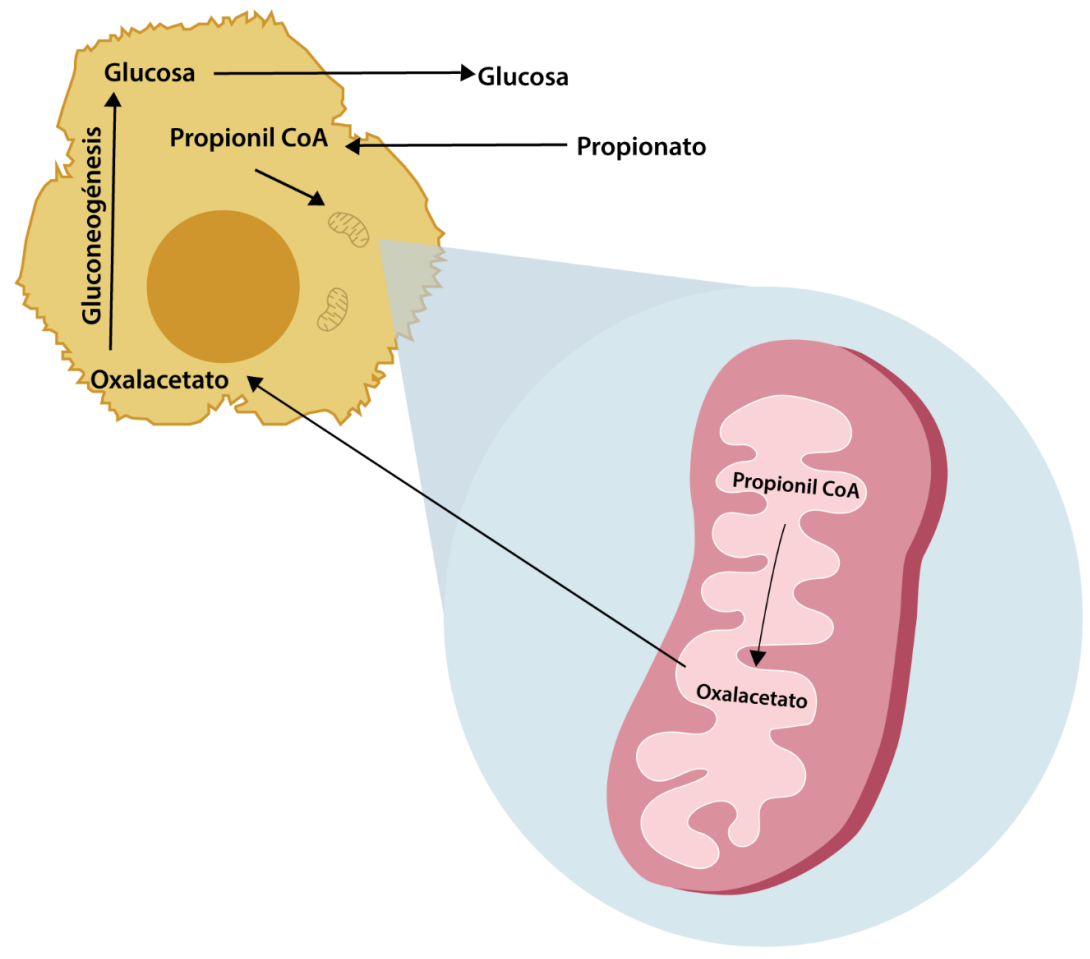

Figura 4. Descripción de la ruta metabólica del propionato, como precursor de oxalacetato y la gluconeogénesis en el hepatocito.

2012). El glicerol resultante de la hidrólisis, por parte de las lipasas, es transportado fuera del adipocito, por la proteína de membrana Acuaporina (Zhang et al. 2019), llega al hígado, en donde ingresa al ciclo gluconeogenico, convertido en dihidroxiacetona fosfato (DHAP), por las enzimas citoplasmáticas glicerol cinasa (Miao et al. 2019) y glicerol fosfato deshidrogenasa (Figura 5) (Wei et al. 2019). La lipolisis es un proceso que demanda gran cantidad de energía, porque la utilización del glicerol depende de la disponibilidad tisular de la enzima glicerol cinasa que, habitualmente, se encuentra en cantidades importantes en hígado, en riñones, en intestino y en glándula mamaria lactante (Murray et al. 2012).

Metabolismo hepático, a través de la beta oxidación de ácidos grasos libres o no esterificados (Figura 5). Una vez adentro del hepatocito, el AGNE sufre un proceso de activación por la proteína de unión a ácidos grasos (FABP) y es transportado a la membrana mitocondrial en la que se encuentra el receptor, denominado CarnitilPalmitoil transferasa-1 (CPT-1), que desplaza el grupo CoA y el ácido graso, se une a la carnitina, con lo cual, puede ingresar a la matriz de la mitocondria. Posteriormente, la Carnitil-Palmitoil transferasa 2 (CPT-2), ubicada en la membrana interna de la mitocondria, captura la molécula de carnitina, adherida al ácido graso y le devuelve un grupo CoA (Bruss, 2008; Murray et al. 2012; Engelking, 2015).

La $\beta$-oxidación de los AGNE's es un proceso cíclico de óxidoreducción y está mediado por las enzimas Acil-CoA deshidrogenasa, Enoil-CoA hidratasa, $\beta$-ketoacil-CoA deshidrogenasa y tiolasa, que dejan como resultado Acetil-CoA, disponible para ser usado en el ciclo de Krebs (Heitmann et al. 1987).

Cuando el Acetil CoA no puede ser utilizado por el ciclo de Krebs, debido a que no hay grandes cantidades de oxalacetato, éste se acumula en la mitocondria, dando lugar a la formación de cuerpos cetónicos: AcAc y $\beta \mathrm{HB}$ (Figura 5) (Bruss, 2008). La acetona, también es considerada un cuerpo cetónico y es formada por la descarboxilación espontánea de AcAc, principalmente, en el tejido pulmonar (Murray et al. 2012). El AcAc y el $\beta H B$ son la principal fuente de energía alterna en todos los tejidos extrahepáticos, principalmente corazón, músculo esquelético, glándula mamaria y riñones.

Metabolismo de los cuerpos cetónicos en los tejidos extrahepáticos. El $\beta \mathrm{HB}$ es el cuerpo cetónico más abundante. Después de ser producido en el epitelio ruminal y en el tejido hepático es liberado del hígado hacía circulación y transportado y oxidado en las mitocondrias de tejidos periféricos (Murray et al. 2012). Este proceso ocurre en la mitocondria, en una serie de reacciones, denominadas de cetolisis (Sangalli et al. 2018). Después del ingreso a la célula el $\beta \mathrm{HB}$ es convertido en AcAc, por la enzima 3- hidroxibutirato deshidrogenasa (BDH); después es convertida en Acetoacetil CoA, por la enzima que limita este proceso, Succinil CoA-Acetoacetato CoA transferasa (Murray et al. 2012). La enzima Acetil transferasa 1 (ACAT1) cliva el AcAc CoA, dejando como resultado, 2 moléculas de Acetil CoA, para ser utilizadas en el ciclo de Krebs (Sangalli et al. 2018). 




Figura 5. Secuencia de la lipolisis y la movilización de AGNE's desde el tejido graso hasta el hepatocito, donde ingresan a la mitocondria por el incremento de la actividad de CPT-1, y son precursores de cantidades excesivas de Acetil CoA, que no puede ingresar al ciclo de Krebs y termina en cetogénesis exacerbada.

Hipercetonemia. Como se dijo anteriormente, el requerimiento energético extra en el posparto, se produce a partir de los cuerpos cetónicos, originados durante la movilización grasa, que en el hígado, por medio de la $\beta$-oxidación, se va a producir mayor cantidad de Acetil CoA. Además, la glucosa obtenida a partir del propionato está destinada a la formación de piruvato, que más tarde va a ser descarboxilado, para la formación de más Acetil CoA. Este exceso en la producción de Acetil CoA, va a ocasionar un aumento en la formación de cuerpos cetónicos, por encima de los niveles basales.

Por otro lado, para la vaca en balance energético negativo, los AGV cetogénicos (Ácido Acético y ácido butírico), que son producidos en mayor proporción por las bacterias del rumen, continúan representando una gran fuente de Acetil-CoA, que no puede entrar a ciclo de Krebs, por deficiencia de oxalacetato y, por consiguiente, sigue contribuyendo a la formación excesiva de cuerpos cetónicos, denominada hipercetonemia.

\section{CONCLUSIÓN}

La circulación plasmática de cuerpos cetónicos, se considera una condición fisiológica durante el BEN del posparto, porque estos son una fuente importante de energía, que las mitocondrias de tejidos extrahepáticos aprovechan, cuando hay suficiente oxalacetato. La disponibilidad de este último, depende del ácido propiónico, producido por las bacterias del rumen, hecho que lo posiciona como importante precursor de gluconeogénesis y ciclo de Krebs en bovinos. La condición, se considera patológica o se define como cetosis, acetonemia o hipercetonemia, en casos donde la fuente de alimento, la cantidad de leche producida, las reservas corporales de lípidos, estados de enfermedad o ayuno prolongado, puedan generar fallas en los mecanismos de adaptación a los requerimientos energéticos del BEN.

Conflictos de interés: El manuscrito fue preparado y revisado con la participación de todos los autores, quienes declaran que no existe ningún conflicto de interés que ponga en riesgo la validez de la información presentada. Financiación: Este artículo de revisión fue financiado por Colciencias y el grupo de investigación BIOGÉNESIS.

\section{REFERENCIAS}

1. AlUWONG, T.; KOBO, P.I.; ABDULLAHI, A. 2010. Volatile fatty acids production in ruminants and the role of monocarboxylate transporters: a review. African Journal of Biotechnology. 9(38):6229-6232.

2. ANTANAITIS, R.; JUOZAITIEN, V.; TELEVI, M.; MALAŠAUSKIEN, D. 2018. Changes in the real-time registration of milk $\beta$-hydroxybutyrate according to stage and lactation number, milk yield, and status of reproduction in dairy cows. Polish J. Veterinary Sciences (Polonia). 21(4):763-768. https://doi.org/10.24425/pjvs.2018.125589

3. ASCHENBACH, J.R.; KRISTENSEN, N.B.; DONKIN, S.S.; HAMMON, H.M.; PENNER, G.B. 2010. Gluconeogenesis in dairy cows: the secret of making sweet milk from sour dough. IUBMB Life (Estados Unidos). 62(12):869-877. https://doi.org/10.1002/iub.400 
4. BALDWIN, R.L. 1998. Use of isolated ruminal epithelial cells in the study of rumen metabolism. The Journal of Nutrition (Inglaterra), 128:293S-296S. https://doi.org/10.1093/ jn/128.2.293s

5. BALDWIN, R.L.; CONNOR, E.E. 2017. Rumen function and development. Veterinary Clinics of North America - Food Animal Practice (Norte América). 33(3):427-439. https:/ / doi.org/10.1016/j.cvfa.2017.06.001

6. BALDWIN, R.L.; JESSE, B.W. 1991. Technical note: isolation and characterization of sheep ruminal epithelial cells. J. Animal Science (Inglaterra), 69(9):3603-3609. https://doi. org/10.2527/1991.6993603x

7. BALDWIN, R.L.; JESSE, B.W. 1996. Propionate modulation of ruminal ketogenesis. J. Animal Science. 74(7):1694-1700. https://doi.org/10.2527/1996.7471694x

8. BRUSS, M.L. 2008. Lipids and ketones. En: Kaneko, J.; Harvey, J.; Bruss, M.L. (eds). Clinical Biochemistry of Domestic Animals. Ed. ElSEVIER (Estados unidos). p.81-115. https://doi.org/10.1016/B978-0-12-370491-7.00004-0

9. CHANDLER, T.L.; PRALLE, R.S.; DÓREA, J.R.R.; POOCK, S.E.; OETZEL, G.R.; FOURDRAINE, R.H.; WHITE, H.M. 2017. Predicting hyperketonemia by logistic and linear regression using test-day milk and performance variables in early-lactation holstein and jersey cows. J. Dairy Science (Estados Unidos). 101(3):2476-2491. https://doi. org/10.3168/jds.2017-13209

10. CHURCH, D.C. 1993. El rumiante: Fisiología Digestiva Y Nutrición. Ed. Acribia S.A (España). 652p.

11. DIJKSTRA, J.; ELLIS, J.L.; KEBREAB, E.; STRATHE, A.B.; LÓPEZ, S.; FRANCE, J.; BANNINK, A. 2012. Ruminal ph regulation and nutritional consequences of low $\mathrm{pH}$. Animal Feed Science and Technology (Estados Unidos). 172(12):22-33. https://doi.org/10.1016/j.anifeedsci.2011.12.005

12. DUFFIELD, T.F.; LISSEMORE, K.D.; MCBRIDE, B.W.; LESLIE, K.E. 2009. Impact of hyperketonemia in early lactation dairy cows on health and production. Journal of Dairy Science (Estados Unidos). 92(2):571-580. https:// doi.org/10.3168/jds.2008-1507

13. EMMANUEL, B. 1980. Oxidation of butyrate to ketone bodies and CO2 in the rumen epithelium, liver, kidney, heart and lung of camel (camelus dromedarius), sheep (ovis aries) and goat (carpa hircus). Comparative Biochemistry and Physiology -- Part B: Biochemistry and molecular biology (Holanda), 65(4):699-704. https://doi.org/10.1016/03050491(80)90182-0
14. EMMANUEL, B. 1981. Further metabolic studies in the rumen epithelium of camel (camelus dromedarius) and sheep (ovis aries). Comparative Biochemistry and Physiology Part B: Comparative Biochemistry. 68(1):155-158. https://doi. org/10.1016/0305-0491(81)90196-6

15. EMMANUEL, B.; MILLIGAN, L.P. 1983. Butyrate: acetoacetylcoa transferase activity in bovine rumen epithelium. Canadian J. Animal Science. 63(1):355-360. https://doi. org/10.4141/cjas83-043

16. ENGELKING, L.R. 2015. Textbook of veterinary physiological chemistry. Ed. Elsevier (Estados Unidos). 786p.

17. FRANKLUNDT, C.V.; GLASS, T.L. 1987. Glucose uptake by the cellulolytic ruminal anaerobe bacteroides succinogenes. J. Bacteriology (Estados Unidos). 169(2):500-506.

18. FRIGGENS, N.C.; BERG, P.; THEILGAARD, P.; KORSGAARD, I.R.; INGVARTSEN, K.L; LØVENDAHL, P.; JENSEN, J. 2007. Breed and parity effects on energy balance profiles through lactation: evidence of genetically driven body energy change. J. Dairy Science (Estados Unidos). 90(11):5291-5305. https://doi.org/10.3168/ jds.2007-0173

19. GARZÓN-AUDOR, A.M.; OLIVER-ESPINOSA, O.J. 2018. Incidencia y prevalencia de cetosis clínica y subclínica en ganado en pastoreo en el altiplano cundiboyacense, Colombia. CES Medicina Veterinaria Y Zootecnia (Colombia). 13(2):121-136. https://doi.org/10.21615/ cesmvz.13.2.3

20. HACKMANN, T.J.; FIRKINS, J.L. 2015. Electron transport phosphorylation in rumen butyrivibrios: unprecedented atp yield for glucose fermentation to butyrate. Frontiers in Microbiology (Estados Unidos). 6(1):1-11. https://doi. org/10.3389/fmicb.2015.00622

21. HACKMANN, T.J.; NGUGI, D.K.; FIRKINS, J.L.; TAO, J. 2017. Genomes of rumen bacteria encode atypical pathways for fermenting hexoses to short- chain fatty acids Tim. Environmental Microbiology (Estados Unidos), $19(11): 4670-4683$. https://doi.org/10.1111/14622920.13929

22. HARFOOT, C.G. 1981. Lipid metabolism in the rumen. En: Lipid metabolism in ruminant animals. Pergamon. p. 21-55.

23. HEITMANN, R.N.; DAWES, D.J.; SENSENIG, S.C. 1987. Hepatic ketogenesis and peripheral ketone body utilization in the ruminant. The Journal of Nutrition (Inglaterra). 117(6):1174-1180. https://doi.org/10.1093/jn/117.6.1174 
24. HERD'T, T.H. 2000. Ruminant adaptation to negative energy balance. Veterinary Clinics of North America: Food Animal Practice (Estados Unidos). 16(2):215-230. https://doi. org/10.1016/s0749-0720(15)30102-x

25. JIANG, W.; PINDER, R.S.; PATTERSON, J.A.; RICKE, S.C. 2014. Sugar phosphorylation activity in ruminal acetogens. J. Environmental Science and Health (Estados Unidos). 18(25):37-41. https://doi.org/10.1080/10934529.2012.66 4998

26. KATO, D.; SUZUKI, Y.; SATOSHI, H.; HAGA, S.; SO, K.; YAMAUCHI, E.; NAKANO, M; ISHIZAKI, H.; CHOI, K.; KATOH, K., ROH, S. 2015. Utilization of digital differential display to identify differentially expressed genes related to rumen development. Animal Science Journal. 87(4):584-590. https://doi.org/10.1111/asj.12448

27. KIRAT, D.; MASUOKA, J.; HAYASHI, H.; IWANO, H.; YOKOTA, H.; TANIYAMA, H.; KATO, S. 2006. Monocarboxylate transporter 1 (mct1) plays a direct role in short-chain fatty acids absorption in caprine rumen. J. Physiology (Estados Unidos). 576(2):635-647. https://doi. org/10.1113/jphysiol.2006.115931

28. KIRAT, D.; MATSUDA, Y.; YAMASHIKI, N.; HAYASHI, H.; KATO, S. 2007. Expression, cellular localization, and functional role of monocarboxylate transporter 4 (mct4) in the gastrointestinal tract of ruminants. Gene (Holanda). 391(1-2):140-149. https://doi.org/10.1016/j. gene.2006.12.020

29. KOHO, N.; MAIJALA, V.; NORBERG, H.; NIEMINEN, M.; PÖSÖ, A.R. 2005. Expression of met1, met2 and mct4 in the rumen, small intestine and liver of reindeer (Rangifer tarandus tarandus L.). Comparative Biochemistry and Physiology - A Molecular and Integrative Physiology (Holanda). 141(1):29-34. https://doi.org/10.1016/j. cbpb.2005.03.003

30. KRISTENSEN, N.B.; HUNTINGTON, G.B.; HARMON, D.L. 2005. Splanchnic carbohydrate and energy metabolism in growing ruminants. In: Burrin D.G.; Mersman H.J. (eds.). Biology of Growing Animals p.405-432. Boston. https:/ / doi.org/10.1016/S1877-1823(09)70024-4

31. LANGIN, D. 2006. Control of fatty acid and glycerol release in adipose tissue lipolysis. Comptes Rendus - Biologies (Francia). 329(8):598-607. https://doi.org/10.1016/j. crvi.2005.10.008

32. LEIGHTON, B.; NICHOLAS, A.R.; POGSON, C.I. 1983. The pathway of ketogenesis in rumen epithelium of the sheep. The Biochemical J. (Inglaterra). 216(3):769-772. https:/ / doi.org/10.1042/bj2160769
33. LIU, L.; ZHUGE, X.; SHIN, H.D.; CHEN, R.R.; LI, J.; DU, G.; CHEN, J. 2015. Improved production of propionic acid in Propionibacterium jensenii via combinational overexpression of glycerol dehydrogenase and malate dehydrogenase from klebsiella pneumoniae. Applied and Environmental Microbiology (Estados Unidos). 81(7):2256-2264. https:/ doi.org/10.1128/AEM.03572-14

34. MAAS, L.K.; GLASS, T.L. 1991. Celobiose uptake by the cellulolytic ruminal anaerobe Fibrobacter (Bacteroides) succinogenes. Can. J. Microbiol. (Canadá). 37(1):141-147. https://doi.org/10.1139/m91-021

35. MADRESEH-GHAHFAROKHI, S.; DEHGHANISAMANI, A.; DEHGHANI-SAMANI, A. 2018. Ketosis (acetonaemia) in dairy cattle farms: practical guide based on importance, diagnosis, prevention and treatments. J. Dairy, Veterinary \& Animal Research (Hungría), 7(6):299-302. https://doi. org/10.15406/jdvar.2018.07.00230

36. MANN, S.; YEPES, F.A.L.; BEHLING-KELLY, E.; MCART, J.A.A. 2017. The effect of different treatments for earlylactation hyperketonemia on blood $\beta$-hydroxybutyrate, plasma nonesterified fatty acids, glucose, insulin, and glucagon in dairy cattle. J. Dairy Science (Estados Unidos), 100(8):6470-6482. https://doi.org/10.3168/jds.2016-12532

37. MATSUMOTO, H.; SASAKI, K.; BESSHO, T.; KOBAYASHI, E.; ABE, T.; SASAZAKI, S.; OYAMA, K.; MANNEN, H. 2012. The snps in the acaca gene are effective on fatty acid composition in Holstein milk. Molecular Biology Reports (Suiza). 39(9):8637-8644. https://doi.org/10.1007/s11033$012-1718-5$

38. MIAO, L.; YANG, Y.; LIU, Y.; LAI, L.; WANG, L.; ZHAN, Y.; YIN, R.; YU, M.; LI, CH.; YANG, X.; GE, C. 2019. Glycerol kinase interacts with nuclear receptor nr4a1 and regulates glucose metabolism in the liver. FASEB J.Official Publication of the Federation of American Societies for Experimental Biology (Estados Unidos). 33(6):6736-6747. https://doi.org/10.1096/fj.201800945RR

39. Millen, D.D.; ARRIGONI, M.D.B.; DIAS, R. 2016. Rumenology. Ed. Springer L. (Brasil). 314p.

40. MURRAY, R.; BENDER, D.; BOTHAM, K.L.; KENNELLY, P.J.; RODWELL, V.W.; WEIL P.A. 2012. Bioquímica ilustrada harper. Ed. McGrawHill (Estados Unidos). 480p.

41. NAKAMURA, S.; HAGA, S.; KIMURA, K.; MATSUYAMA, S. 2018. Propionate and butyrate induce gene expression of monocarboxylate transporter 4 and cluster of differentiation 147 in cultured rumen epithelial cells derived from preweaning dairy calves. Journal of Animal Science (Inglaterra). 96(11):4902-4911. https://doi.org/10.1093/ jas/sky334 
42. NIELSEN, L.; GONZALEZ-GARCIA, R.; MARCELLIN, E.; NAVONE, L.; STOWERS, C.; MCCUBBIN, T. 2017. Microbial propionic acid production. Fermentation (Estados Unidos). 3(2):21. https://doi.org/10.3390/ fermentation3020021

43. NOCEK, J.E.; HERBEIN, J.H.; POLAN, C.E. 1980. Influence of ration physical form, ruminal degradable nitrogen and age on rumen epithelial propionate and acetate transport and some enzymatic activities. The J. Nutrition (Inglaterra). 110(12):2355-2364. https://doi.org/10.1093/ jn/110.12.2355

44. PABON, M. 2004. Notas de clase. Bioquímica ruminal. Ed. Universidad Nacional de Colombia (Bogotá D.C). 50p.

45. PRATAMA, R.; ARTIKA, I.M.; CHAIDAMSARI, T.; SUGIARTI, H.; PUTRA, S.M. 2014. Isolation and molecular cloning of cellulase gene from bovine rumen bacteria. Current Biochemistry (Indonesia). 1(1):29-36. https://doi. org/10.29244/cb.1.1.29-36

46. PRATTI DANIEL, J.L.; RESENDE JÚNIOR, J.C. 2012. Absorção e metabolismo de ácidos graxos voláteis pelo rúmen e omaso. Ciencia E Agrotecnologia (Brasil). 36(1):9399. https://doi.org/10.1590/S1413-70542012000100012

47. PRAT'TI DANIEL, J.L.; RESENDE JÚNIOR, J.C.; CRUZ, F.J. 2006. Participação do ruminoretículo e omaso na superfície absortiva total do proventrículo de bovinos. Brazilian J. Veterinary Research and Animal Science (Brasil). 43(5):688-694. https://doi.org/10.11606/issn.1678-4456. bjvras.2006.26579

48. RATANAKHANOKCHAI, K.; WAEONUKUL, R.; PASON, P.; TACHAAPAIKOON, C.; KYU, K.L.; SAKKA, K.; KOSUGI, A.; MORI, Y. 2013. Paenibacillus curdlanolyticus strain b-6 multienzyme complex: a novel system for biomass utilization. En: Matovic, M.D (ed.). Biomass Now - Cultivation and Utilization. IntechOpen https://doi. org $/ 10.5772 / 51820$

49. SANGALLI, J.; SAMPAIO, R.V.; DEL COLLADO, J.; COELHO DA SILVEIRA, J.; CAMARA DE BEM, T.H.; PERECIN, F.; SMITH, L.C.; VIEIRA MEIRELLES, F. 2018. Metabolic gene expression and epigenetic effects of the ketone body $\beta$-hydroxybutyrate on h3k9ac in bovine cells, oocytes and embryos. Scientific Reports (Estados Unidos). 8(1):1-18. https://doi.org/10.1038/s41598-018-31822-7

50. SMITH, B.P. 2013. Large animal internal medicine. Eds. Elsevier (Estados Unidos). 1661p.

51. STORM, A.C.; KRISTENSEN, N.B.; HANIGAN, M.D. 2012. A model of ruminal volatile fatty acid absorption kinetics and rumen epithelial blood flow in lactating holstein cows. J. Dairy Science (Estados Unidos). 95(6):2919-2934. https:/ / doi.org/10.3168/jds.2011-4239

52. VAIDYA, J.D.; HORNUNG, B.V.H.; SMIDT, H.; EDWARDS, J.E.; PLUGGE, C.M. 2019. Propionibacterium ruminifibrarum sp. nov., isolated from cow rumen fibrous content. Internal J. Systematic and Evolutionary Microbiology (Inglaterra). 69(8):2584-2590. https://doi.org/10.1099/ijsem.0.003544

53. VAN LINGEN, H.J.; PLUGGE, C.M.; FADEL, J.G.; KEBREAB, E. 2016. Thermodynamic driving force of hydrogen on rumen microbial metabolism: a theoretical investigation. PLoS ONE (Estados Unidos). 11(10):1-18. https://doi.org/10.1371/journal.pone.0161362

54. VITAL, M.; CHUANG HOWE, A.; TIEDJE, J.M. 2014. Revealing the bacterial butyrate synthesis pathways by analyzing (Meta) Genomic Data. American Society for Microbiology (Estados Unidos). 5(2):1-11. https://doi. org/10.1128/mBio.00889-14

55. WANG, L.; ZHANG, G.; LI, Y.; ZHANG, Y. 2020. Effects of high forage/concentrate diet on volatile fatty acid production and the microorganisms involved in $\mathrm{vfa}$ production in cow rumen. Animals (Inglaterra), 10(2):223. https://doi.org/10.3390/ani10020223

56. WATFORD, M.; HOD, Y.; CHIAO, Y.; UTTER, F.; HANSON, R.W. 1981. The unique role of the kidney in gluconeogenesis in the chicken. The J. Biological Chemistry (Estados Unidos). 256(19):10023-10027. Disponible desde Internet en: https://www.jbc.org/content/256/19/10023.long

57. WEBSTER, L.T.; GEROWIN, J.L.; RAKITA, L. 1965. Purification and characteristics of a butyryl coenzime a synthetase from bovine heart mitocondria. The Journal of Biological Chemistry (Estados Unidos). 240(1):29-33.

58. WEI, Y.; LI, X.; ZHANG, D.; YONGFENG, L. 2019. Comparison of protein differences between high- and lowquality goat and bovine parts based on iTRAQ technology. Food Chemistry (Holanda). 289(3):240-249. https://doi. org/10.1016/j.foodchem.2019.03.052

59. WEIGAND, E; YOUNG, J.W.; MCGILLIARD, A.D. 1975. Volatile fatty acid metabolism by rumen mucosa from cattle fed hay or grain. J. Dairy Science (Estados Unidos). 58(9):1294-1300. https://doi.org/10.3168/jds.s00220302(75)84709-6

60. WHITE, D.; DRUMMOND, J.; FUQUA, C. 2012. The physiology and biochemistry of prokaryotes. Eds. Oxford University Press (Estados Unidos). 632p. 
61. WHITE, H.M.; KOSER, S.L.; DONKIN, S.S. 2012. Gluconeogenic enzymes are differentially regulated by fatty acid cocktails in madin-darby bovine kidney cells 1 . J. Dairy Science (Estados Unidos). 95(3):1249-1256. https:/ / doi.org/10.3168/jds.2011-4644

62. WONGKITTICHOTE, P.; MEW, N.A.; CHAPMAN, K.A. 2017. Propionyl-CoA carboxylase-a review. Molecular genetics and metabolism. 122(4):145-152. https://doi. org/10.1016/j.ymgme.2017.10.002

63. XIANG, E.; HUTTON ODDY, V.; ARCHIBALD, A.L.; VERCOE, P.E.; DALRYMPLE, B.P. 2016. Epithelial, metabolic and innate immunity transcriptomic signatures differentiating the rumen from other sheep and mammalian gastrointestinal tract tissues. PeerJ (Estados Unidos). 4:e1762. https://doi.org/10.7717/peerj.1762
64. XU, S.; WU, Z.; ZOU, Y.; LI, S.; CAO, Z. 2017. Evaluation of a hand-held meter to detect subclinical ketosis in dairy cows. Advances in Dairy Research (Estados Unidos). 5(2):2-5. https://doi.org/10.4172/2329-888x.1000173

65. XU, W.; VERVOORT, J.; SACCENTI, E.; VAN HOEIJ, R.; KEMP, B.; VAN KNEGSEL, A. 2018. Milk metabolomics data reveal the energy balance of individual dairy cows in early lactation. Scientific Reports (Estados Unidos). 8(1):1 11. https://doi.org/10.1038/s41598-018-34190-4

66. ZHANG, J.; TAN, J.; ZHANG, C.; WANG, Y.; CHEN, X.; LEI, C.; CHEN, H.; FANG, X. 2019. Research on associations between variants and haplotypes of aquaporin 9 (aqp9) gene with growth traits in three cattle breeds. Animal Biotechnology (Inglaterra). https://doi.org/10.1080/1049 5398.2019.1675681 